\title{
Diagnostic and prognostic value of placental growth factor serum concentration in clear cell renal cell carcinoma
}

\author{
Marcela Cechova ${ }^{a}$, Matus Chocholaty ${ }^{a}$, Marek Babjuka ${ }^{\mathrm{a}}$,Tomas Zima ${ }^{\mathrm{b}}$, Klara Havlova ${ }^{\mathrm{a}}$, Marketa Koldovaa ${ }^{\mathrm{a}}$, Marek Schmidta \\ Marta Kalousovab
}

Background and Aim: Placental Growth Factor (PIGF) plays a crucial role in angiogenesis and was identified as a potential prognostic biomarker in various types of cancer. Therefore, we evaluated the diagnostic accuracy and prognostic value of PIGF serum concentration in patients with clear cell renal cell carcinoma (ccRCC).

Patients and Methods: A total of 49 patients subjected to partial or radical nephrectomy for ccRCC [localized without relapse (IccRCC; $n=31)$, localized with later relapse $(r c R C C ; n=8)$, primary metastatic cancer (mccRCC; $n=10)$; median of follow-up 4.4 years] were enrolled in a prospective study to assess the significance of PIGF serum concentration. PIGF was measured prior to surgery and 3 months postoperatively. Our control group consisted of 38 healthy subjects. Results: PIGF serum concentration was significantly higher in ccRCC compared to controls $(P=0.002)$. The cut-off value of PIGF concentration for the risk of $c c R C C$ was determined at $12.71 \mathrm{pg} / \mathrm{mL}$ ( $A U C=0.729 ; P=0.0001$ ). Prior to surgery, among $\mathrm{ccRCC}$ subgroups, significantly higher PIGF concentration was detected in mccRCC compared to IccRCC $(P=0.002)$. Postoperatively, we observed a tendency to higher PIGF serum concentration in rccRCC compared to IccRCC subgroup, however without significance $(P=0.17)$. The cut-off value for the risk of relapse was $11.41 \mathrm{pg} / \mathrm{mL}(A \cup C=0.792$; $P=0.0003$ ). In subjects with localized ccRCC with PIGF concentration below $11.41 \mathrm{pg} / \mathrm{mL}$ 3-years cancer specific survival was $93 \%$ compared to $61 \%$ in subject with concentration above the cut-off value ( $P=0.018)$.

Conclusion: Based on our findings, PIGF serum concentration seems to be a useful biomarker in diagnostics and prediction of prognosis in ccRCC.

Key words: placental growth factor, PIGF, clear cell renal cell carcinoma, biomarker, diagnosis, prognosis

Received: May 1, 2020; Revised: November 9, 2020; Accepted: January 8, 2021; Available online: February 22, 2021

https://doi.org/10.5507/bp.2021.003

(c) 2021 The Authors; https://creativecommons.org/licenses/by/4.0/

${ }^{a}$ Department of Urology, University Hospital Motol and $2^{\text {nd }}$ Faculty of Medicine, Charles University, Prague, Czech Republic bInstitute of Medical Biochemistry and Laboratory Diagnostics, General University Hospital and $1^{\text {st }}$ Faculty of Medicine, Charles University, Prague, Czech Republic

Corresponding author: Marta Kalousova, e-mail: marta.kalousova@lf1.cuni.cz

\section{INTRODUCTION}

Worldwide, renal cell carcinoma (RCC) is the sixth most frequently diagnosed cancer in men and eighth in women, accounting for $5 \%$ respectively $3 \%$ of all carcinomas with the highest incidence in the Western countries ${ }^{1}$. In 2018 in Europe new cases of RCC were estimated to occur in 136,500 patients resulting in 54,700 deaths ${ }^{2}$.

Although most detected RCCs are incidentally diagnosed small tumours, locally advanced disease is still found in a significant proportion of patients with up to one third presenting with distant metastasis at the time of diagnosis $^{3}$. Moreover, about $20-40 \%$ patients experience recurrence after surgical treatment of localized disease $e^{4}$. The introduction of targeted agents has considerably improved the prognosis of patients with metastatic disease, yet, median survival is beyond two years 5 .

Despite improvements in diagnostics and treatment, RCC still remains among urological malignancy with the highest mortality. Although several risk models for prediction of recurrence have been introduced, the individual course of diseases is difficult to predict. Biomarkers can improve the diagnostics of RCC, and even more can pro- vide additional predictive accuracy in identifying patients with higher risk of recurrence which would enable treatment individualization and improvement of patients' prognosis. Therefore, investigation of potential biomarkers is needed.

Cancer-related angiogenesis is crucial for tumour growth and progression, and it is regulated by various tumour cell produced growth factors ${ }^{6}$. Placental growth factor (PlGF) belongs to vascular endothelial growth factor (VEGF) family, which is the most important trigger of angiogenesis and endothelial cell growth. PIGF stimulates angiogenesis specifically targeting VEGF receptor 1 (Flt-1) and co-receptor neuropilin (NRP1). Originally PIGF was described in human placenta and it is highly expressed throughout pregnancy ${ }^{7}$. In healthy subjects PIGF serum levels are typically low but increase under pathological conditions such as ischemia, inflammation or tumour growth ${ }^{8,9}$. Overexpression of PIGF has been described in several types of carcinomas. Furthermore, PIGF was identified as a potential prognostic biomarker of cancer progression as its higher expression correlates with advanced tumour stage, hypervascularity, presence of metastasis and shorter patient surviva ${ }^{10-15}$. However, there 
is only a limited number of studies assessing the role and significance of PIGF in RCC ( ref. $^{16,17}$ ).

The present study aimed to evaluate PlGF serum concentration in patients suffering from clear cell renal cell carcinoma (ccRCC), and furthermore, to explore its diagnostic accuracy and prognostic value.

\section{PATIENTS AND METHODS}

\section{Subjects}

Into this prospective study we included 49 individuals (30 men, 19 women, mean age $62.9 \pm 10.6$ years) who were subjected to radical or partial nephrectomy due to ccRCC from June 2011 to June 2013. In all resected tumour specimens negative surgical margins were described. For further comparison ccRCC subjects were classified in three subgroups: subjects with localized ccRCC without further relapse (lccRCC, $n=31$ ), subjects with localized ccRCC with relapse 6 to 18 months following surgical treatment (rccRCC, $n=8$ ) and subjects with primary metastatic carcinoma (mccRCC, $n=10$ ). The staging of ccRCC was performed following the 2009 TNM classification system, Fuhrman Nuclear Grading System was used for evaluation of the tumour grade. Surveillance after partial or radical nephrectomy was performed in adherence to the EAU Guidelines for Renal Cell Carcinoma ${ }^{18}$. Median of follow-up of our study group was 4.4 years. During the follow-up relapse occurred in 8 subjects (rccRCC subgroup) and 17 subjects died. Table 1 describes demographic and clinicopathological parameters of ccRCC cases.

Thirty-eight matched for age and sex healthy subjects with no history of any malignant disease formed the control group. In all, basic uro-oncological screening including ultrasound, urine cytology and measurement of PSA levels in men was performed.

The study was conducted in adherence to ethical guidelines and approved by the institutional review board and ethics committee. A written informed consent was obtained from all participants prior to entering the study.

Table 1. Parameters of ccRCC patients.

\begin{tabular}{lc}
\hline Number of patients (men/women) & $49(30 / 19)$ \\
Age (years) & $62.9 \pm 10.6$ \\
\hline Stage & $23(46.9 \%)$ \\
I & $1(2 \%)$ \\
II & $15(30.6 \%)$ \\
III & $10(20.4 \%)$ \\
IV & \\
\hdashline Grade & $10(20.4 \%)$ \\
G1 & $31(63.3 \%)$ \\
G2 & $5(10.2 \%)$ \\
G3 & $3(6.1 \%)$ \\
G4 & \\
\hline
\end{tabular}

ccRCC: clear cell renal cell carcinoma

\section{Blood samples}

In all individuals P1GF serum concentration was measured prior to surgical treatment. 3 months postoperatively blood samples were collected from patients with localized disease (lccRCC and rccRCC subgroups). Blood samples were obtained after overnight fast by puncture via cubital vein, at the same time with blood collection for routine control examinations. For PlGF analysis blood was centrifuged for $10 \mathrm{~min}$ at $3000 \mathrm{rpm}$ and serum was stored at $-80{ }^{\circ} \mathrm{C}$ until analysis.

\section{Biomarker analysis}

PlGF serum concentration was assessed with the enzyme-linked immunosorbent assay (ELISA) using standard kits (R\&D Systems, Minneapolis, MN, USA) following the manufacturers' instructions. Results are expressed in picograms per millilitre $(\mathrm{pg} / \mathrm{mL})$.

\section{Statistical analysis}

Data are presented as the means \pm standard deviation (SD) for continuous variables and percentages for categorical variables. For continuous variables the difference between subgroups was analysed by one-way ANOVA or Mann-Whitney U test, as appropriate. The repeated measures ANOVA test was used for the analysis of continuous variables over time. The Receiver Operating Characteristics (ROC) curves were created and relative potential of PIGF to identify ccRCC was specified with calculation of area under the ROC curve (AUC). KaplanMeier method was used for survival analysis and longrank test was performed to compare survival between subgroups. Univariate Cox proportional hazards regression analyses were applied for assessment of PlGF serum concentration as a potential predictor of cancer specific and overall survival. Statistical significance was set at $P<0.05$. Data analyses were carried out using MedCalc for Windows, version 13.0 (MedCalc Software, Ostend, Belgium).

\section{RESULTS}

\section{Diagnostic accuracy of PIGF in ccRCC}

PlGF serum concentration in ccRCC subjects $(16.1 \pm 10.5 \mathrm{pg} / \mathrm{mL})$ was significantly higher compared to healthy controls $(10.5 \pm 2.4 \mathrm{pg} / \mathrm{mL})(P=0.002)$. The cut-off value of PlGF serum concentration for the risk of ccRCC was determined at $12.71 \mathrm{pg} / \mathrm{mL}$ with AUC 0.729 , providing the specificity of $84.21 \%$ and sensitivity of $61.22 \%$ ( $P=0.0001)$ (Fig 1). Prior to surgical treatment, a significant difference in PlGF serum concentration was found between lccRCC $(13.1 \pm 5.1 \mathrm{pg} / \mathrm{mL})$ and mccRCC $(25.8 \pm 18.1 \mathrm{pg} / \mathrm{mL})$ subgroups $(P=0.002)$. However, we found no statistically significant difference between rccRCC $(15.9 \pm 6.9 \mathrm{pg} / \mathrm{mL})$ and $1 \mathrm{ccRCC}$ or mccRCC subgroups, respectively. PlGF serum concentration was not found to correlate with tumour stage (T1-T2 vs T3-T4, $P=0.76)$ or Fuhrman's grade (G1-G2 vs G3-G4, $P=0.07)$. 


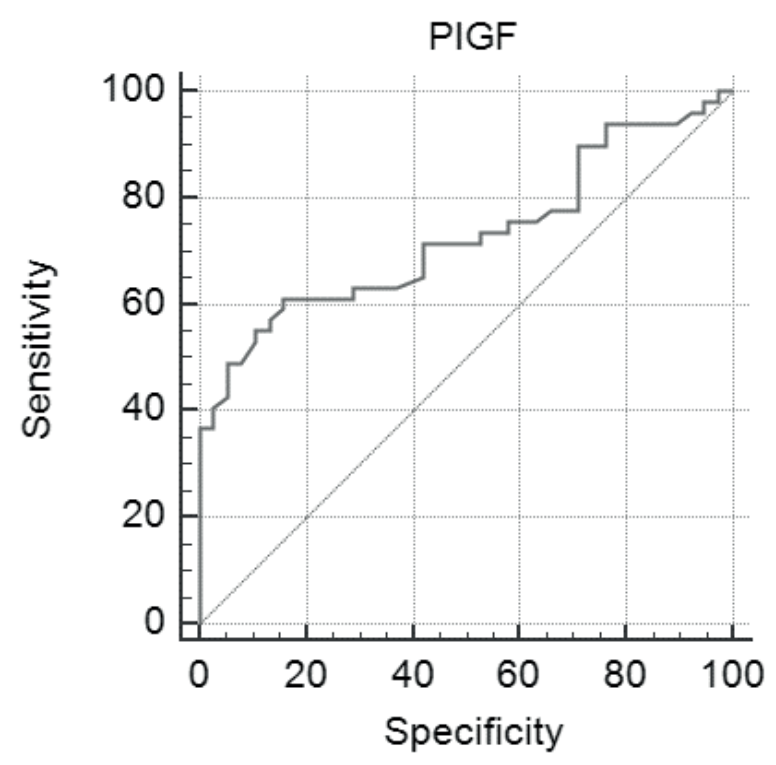

Fig. 1. Cut-off value of PIGF serum concentration for the risk of ccRCC.

Cut-off value of PlGF serum concentration for the risk of ccRCC $>12.71 \mathrm{pg} / \mathrm{mL}$ (AUC 0.729 , specificity $84.21 \%$, sensitivity $61.22 \%, P=0.0001)$.

PIGF: placental growth factor, ccRCC: clear cell renal cell carcinoma, AUC: area under the curve

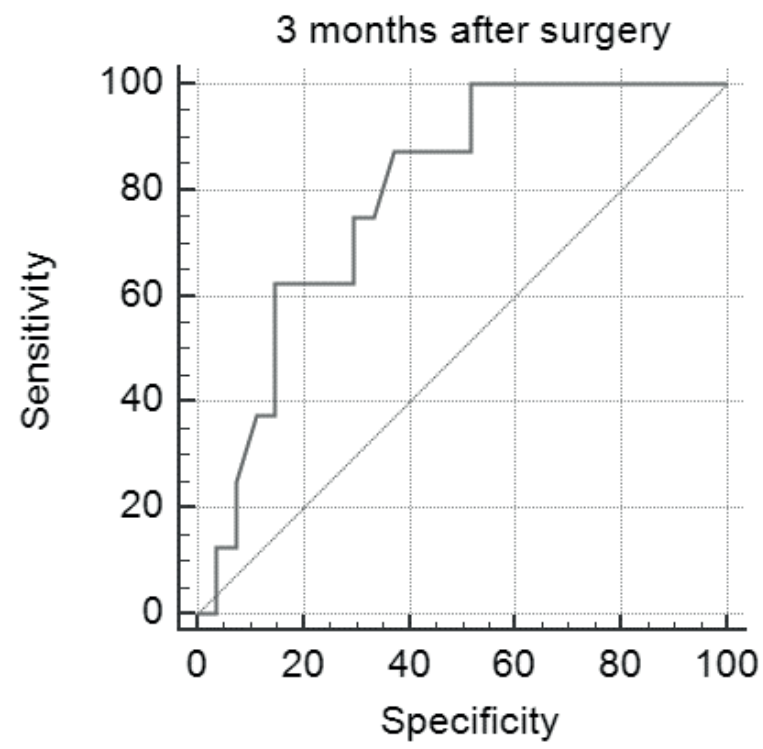

Fig. 2. Cut-off value of PlGF serum concentration for the risk of ccRCC recurrence.

Cut-off value of PIGF serum concentration for the risk of ccRCC recurrence $>11.41 \mathrm{pg} / \mathrm{mL}$ (AUC 0.792 , specificity $62.96 \%$, sensitivity $87.5 \%, P=0.0003$ ).

PLGF: placental growth factor, ccRCC: clear cell renal cell carcinoma, AUC: area under the curve

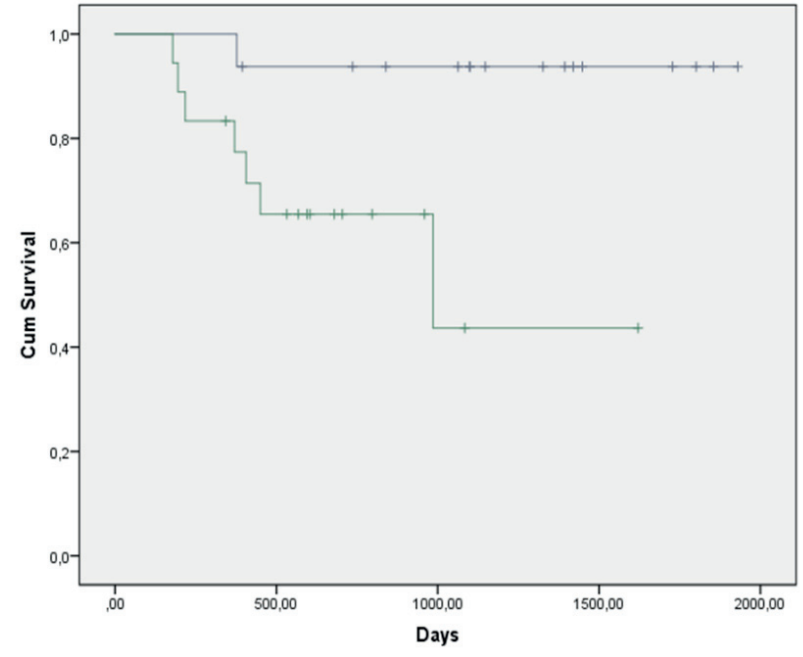

Fig. 3. Kaplan-Maier curves of 3-years cancer specific survival (CSS).

Kaplan-Maier curves of 3-years cancer specific survival (CSS). Upper line - patients with PIGF serum concentration <11.41 $\mathrm{pg} / \mathrm{mL}$ 3-years CSS 93\%, lower line - patients with PIGF serum concentration $>11.41 \mathrm{pg} / \mathrm{ml}$ 3-years CSS $61 \%$.

PIGF: placental growth factor, CSS: cancer specific survival.

\section{Prognostic value of PIGF in ccRCC}

Three months postoperatively, higher PlGF serum concentration in rccRCC $(14.87 \pm 2.96 \mathrm{pg} / \mathrm{mL})$ compared to lccRCC $(12.1 \pm 5.4 \mathrm{pg} / \mathrm{mL})$ subgroup was found, but the difference was not statistically significant $(P=0.17)$. Table 2 depicts all PIGF serum concentrations.

However, the cut-off value for the risk of relapse was determined at $11.41 \mathrm{pg} / \mathrm{mL}$ with AUC 0.792, providing the specificity of $62.96 \%$ and sensitivity of $87.5 \%$ $(P=0.0003)$ (Fig 2).

3-years cancer specific survival in localized ccRCC subjects (1ccRCC and rccRCC subgroup) with PlGF serum concentration below the cut-off $(<11.41 \mathrm{pg} / \mathrm{mL})$ was 93\% compared to $61 \%$ in subjects with PlGF serum concentration above the established cut-off for the risk of relapse (>11.41 pg/mL) (HR 7.9967, CI 1.9938 to 32.0733 , $P=0.018)$ (Fig 3).

\section{DISCUSSION}

Angiogenesis is essential for tumour growth and is assumed to be a key factor of cancer progression and development of metastasis ${ }^{6}$. Among various angiogenic factors VEGF is the most powerful and has been extensively studied over the last years. PIGF is a member of VEGF family and its overexpression is associated predominantly with pathological angiogenesis. Increased PIGF concentrations have been described in several conditions including carcinogenesis. Additionally, several studies imply that higher circulating levels of PIGF correlate with cancer aggressiveness. Therefore, PIGF was identified to be a prognostic marker of cancer progression of many 
Table 2. PIGF serum concentrations.

\begin{tabular}{|c|c|c|c|c|c|}
\hline \multirow[t]{3}{*}{$\begin{array}{l}\text { PIGF }(\mathrm{pg} / \mathrm{mL}) \\
\text { Preoperative }\end{array}$} & & $\begin{array}{c}\text { ccRCC } \\
16.1 \pm 10.5\end{array}$ & & $\begin{array}{l}\text { Controls } \\
10.5 \pm 2.4\end{array}$ & $\begin{array}{l}P \\
P=0.002\end{array}$ \\
\hline & & groups of $\mathrm{ccl}$ & & & \\
\hline & lccRCC & recRCC & mecRCC & & \\
\hline Preoperative & $13.1 \pm 5.1$ & $15.9 \pm 6.9$ & $25.8 \pm 18.1$ & $\cdots$ & lccRCC vs mecRCC $P=0.002 *$ \\
\hline 3 months after surgery & $12.1 \pm 5.4$ & $14.87 \pm 2.96$ & - & - & $P=0.17$ \\
\hline
\end{tabular}

*Preoperatively, PIGF serum concentration significantly higher in mccRCC compared to lccRCC $(P=0.002)$. No statistically significant difference between lccRCC vs recRCC and rccRCC vs mecRCC.

PIGF: placental growth factor, ccRCC: clear cell renal cell carcinoma, lccRCC: localized ccRCC without further relapse, rccRCC: localized ccRCC with later relapse, mccRCC: metastatic ccRCC.

tumours including breast, ovarian, gastric, colorectal, hepatocellular, lung carcinoma or malignant pleural mesothelioma ${ }^{10-15,19-22}$.

Although the role of PlGF in diagnostics and estimation of prognosis has been studied in various tumours, little in known regarding this angiogenic factor in RCC. Since, hypervascularization is a common feature among various solid tumours as well as RCC, production of angiogenic factors in kidney cancer cells have been presumed. Takahashi et al. (ref. ${ }^{16}$ ) as first proved elevated expression of VEGF and PIGF in hypervascular RCC tissue with no detection of PIGF in normal kidney tissue. Matsumoto et al. (ref. ${ }^{17}$ ) analysed P1GF serum levels and its correlation with clinical features of RCC and described association between PlGF concentration and histological grade and tumour vascularity. Furthermore, proposed a prognostic significance of PIGF in RCC.

The aim of the present study was to explore the significance of PIGF serum concentration in patients with ccRCC, the predominant histological subtype of RCC. In the first place, we evaluated the diagnostic significance of PlGF serum concentration. Consistent with previous studies we confirmed significantly higher PIGF serum concentration in ccRCC patients compared to healthy controls. The cut-off value for PIGF serum concentration for the risk of ccRCC was established at $12.71 \mathrm{pg} / \mathrm{mL}$, reaching the specificity of $84.21 \%$ and sensitivity of $61.22 \%$ with AUC 0.729. Based on our results, PlGF serum concentration can be considered as a diagnostic biomarker for detection of ccRCC. However, with respect to AUC most probably as a component of multi-marker diagnostic test rather than a single-marker test.

In our study group PlGF serum concentration was not found to correlate with tumour stage and Fuhrman 's grade. Although, a trend towards higher PlGF serum concentration was observed in ccRCC with more aggressive grade, the results were not significant (G1-G2 vs G3-G4, $P=0.07$ ). This could be explained by the small number of patients and a higher proportion of lower stage and grade tumours.

Secondly, we assessed the potential value of P1GF serum concentration in prediction of relapse and prognosis in patients with localized disease. Postoperatively, we observed a tendency to higher PlGF serum concentration in rccRCC compared to lccRCC. The cut-off value of PIGF serum concentration for the risk of relapse was determined at $11.41 \mathrm{pg} / \mathrm{mL}$ and provided the specificity of
$62.96 \%$ and sensitivity of $87.5 \%$ with AUC 0.792. 3-years cancer specific survival in patients with localized disease with PIGF serum concentration below this cut-off was $93 \%$ compared to only to $61 \%$ with concentration above the cut-off. According to these findings, we presume P1GF serum concentration can be useful in identifying patients with higher risk of recurrence. Even more, PlGF serum concentration can be considered as a potential prognostic biomarker in ccRCC.

Based on previously published studies we assumed higher PlGF serum concentrations in ccRCC patients compared to healthy individuals. Still, the primary goal of our study was to assess whether higher serum concentrations are of clinical importance in diagnosis and estimation of prognosis in ccRCC. Therefore, we did not examine expression of PIGF in tumour tissue. Main limit of our study was a small number of patients in each subgroup. PlGF was shown to have a significant position in gynaecological non-oncological testing (early evaluation of the risk of pre-eclampsia in pregnant women), resulting in broadening and availability of diagnostic technologies of the marker. Combined with the fact, that ccRCC does not have a suitable biomarker, P1GF could, based on our results, show to be a promising one. Though, further studies on larger patient cohorts are necessary to confirm this premise.

\section{CONCLUSION}

We can conclude that PIGF can be a useful biomarker in diagnostics and prediction of prognosis in subjects with ccRCC. Our results show higher PIGF serum concentration in ccRCC patients compared to healthy controls with the cut-off value for the risk of ccRCC at $12.71 \mathrm{pg} / \mathrm{mL}$. Concomitantly PlGF seems to be a predictor of relapse and prognosis in patients with localized disease with the cut-off value at $11.41 \mathrm{pg} / \mathrm{mL}$. In these patients with PIGF serum level below the cut-off, 3-years cancer specific survival was $93 \%$ in comparison to $61 \%$ for those with level above the cut-off.

Acknowledgements: Results of the study were presented at EAU 17th Central European Meeting in Plzeň, Czech Republic, 2017. Abstract named "Placental growth factor - a biomarker for diagnosis, follow-up and prognosis in renal cell cancer" by authors Chocholaty M, Cechova M, 
Babjuk M, Havlova K, Koldova M, Schmidt M, Kalousova $\mathrm{M}$ and Zima T is available at Eur Urol Suppl 2017; 16: e2845. The present study was supported by research grants MH CZ - DRO VFN 64165, Progres Q25 and MH CZ 00064203. For technical support the authors are grateful to Mrs. Dita Hudcová, Institute of Medical Biochemistry and Laboratory Diagnostics, Prague, Czech Republic.

Author contributions: MC: sample and clinical data collection, data analysis, manuscript writing; MCh: sample and clinical data collection, conceptualization, formal and statistical analysis, critical reading and review of the manuscript; $\mathrm{MB}$ : expert opinion and supervision of the clinical part of the study, data interpretation, critical reading and review of the manuscript; TZ: expert opinion and supervision of the laboratory part of the study, data interpretation, critical reading and review of the manuscript; $\mathrm{KH}, \mathrm{MKo}, \mathrm{MS}$ : sample and clinical data collection; MKa: study design, biochemical analysis, data interpretation, critical reading and review of the manuscript.

Conflicts of interest statement: The authors state that there are no conflicts of interest regarding the publication of this article.

\section{REFERENCES}

1. Capitanio U, Bensalah K, Bex A, Boorjian SA, Bray F, Coleman J, Gore JL, Sun M, Wood C, Russo P. Epidemiology of Renal Cell Carcinoma. Eur Urol 2019;75(1):74-84

2. Ferlay J, Colombet M, Soerjomataram I, Dyba T, Randi G, Bettio M, Gavin A, Visser O, Bray F. Cancer incidence and mortality patterns in Europe: Estimates for 40 countries and 25 major cancers in 2018. Eur J Cancer 2018;103:356-387.

3. Gupta K, Miller JD, Li JZ, Russell MW, Charbonneau C. Epidemiologic and socioeconomic burden of metastatic renal cell carcinoma (mRCC): a literature review. Cancer Treat Rev 2008;34(3):193-205.

4. Speed JM, Trinh QD, Choueiri TK, Sun M. Recurrence in Localized Renal Cell Carcinoma: a Systematic Review of Contemporary Data. Curr Urol Rep 2017;18(2):15

5. Gore ME, Larkin JM. Challenges and opportunities for converting renal cell carcinoma into a chronic disease with targeted therapies. Br J Cancer 2011;104(3):399-406.

6. Carmeliet P. Angiogenesis in life, disease and medicine. Nature 2005;438(7070):932-6.

7. Maglione D, Guerriero V, Viglietto G, Delli-Bovi P, Persico MG Isolation of a human placenta CDNA coding for a protein related to the vascular permeability factor. Proc Natl Acad Sci U S A $1991 ; 88(20): 9267-71$.
8. Autiero M, Luttun A, Tjwa M, Carmeliet P. Placental growth factor and its receptor, vascular endothelial growth factor receptor-1: novel targets for stimulation of ischemic tissue revascularization and inhibition of angiogenic and inflammatory disorders. J Thromb Haemost 2003;1(7):1356-70.

9. Fischer C, Mazzone M, Jonckx B, Carmeliet P. FLT1 and its ligands VEGFB and PIGF: drug targets for anti-angiogenic therapy? Nat Rev Cancer 2008;8(12):942-56.

10. Parr C, Watkins G, Boulton M, Cai J, Jiang WG. Placenta growth factor is over-expressed and has prognostic value in human breast cancer. Eur J Cancer 2005;41(18):2819-27.

11. Song N, Liu H, Ma X, Zhang S. Placental growth factor promotes metastases of ovarian cancer through MiR-543-regulatedMMP7. Cell Physiol Biochem 2015;37(3):1104-12.

12. Chen CN, Hsieh FJ, Cheng YM, Cheng WF, Su YN, Chang KJ, Lee PH. The significance of placenta growth factor in angiogenesis and clinical outcome of human gastric cancer. Cancer Lett 2004;213(1):73-82.

13. Wei SC, Tsao PN, Yu SC, Shun CT, Tsai-Wu JJ, Wu CH, Su YN, Hsieh FJ, Wong JM. Placenta growth factor expression is correlated with survival of patients with colorectal cancer. Gut 2005;54(5):666-72.

14. Ho MC, Chen CN, Lee H, Hsieh FJ, Shun CT, Chang CL, Lai YT, Lee $\mathrm{PH}$. Placenta growth factor not vascular endothelial growth factor $\mathrm{A}$ or $C$ can predict the earlyrecurrence after radical resection of hepatocellular carcinoma. Cancer Lett 2007;250(2):237-49.

15. Soukup V, Čapoun O, Pešl M, Sobotka R, Vávřová L, Hanuš T, Zima T, Kalousová M. Placental Growth Factor in Bladder Cancer Compared to the Diagnostic Accuracy and Prognostic Performance of Vascular Endothelial Growth Factor A. Anticancer Res 2018;38(1):239-246.

16. Takahashi A, Sasaki H, Kim SJ, Tobisu K, Kakizoe T, Tsukamoto T, Kumamoto $Y$, Sugimura T, Terada M. Markedly increased amounts of messenger RNAs for vascular endothelial growth factor and placenta growth factor in renal cell carcinoma associated with angiogenesis. Cancer Res 1994;54(15):4233-7.

17. Matsumoto K, Suzuki K, Koike H, Okamura K, Tsuchiya K, Uchida T, Takezawa Y, Kobayashi M, Yamanaka H. Prognostic significance of plasma placental growth factor levels in renal cell cancer: an association with clinical characteristics and vascular endothelial growth factor levels. Anticancer Res 2003;23(6D):4953-8.

18. Ljungberg B, Bensalah K, Canfield S, Dabestani S, Hofmann F, Hora M, Kuczyk MA, Lam T, Marconi L, Merseburger AS, Mulders P, Powles T, Staehler M, Volpe A, Bex A. EAU guidelines on renal cell carcinoma: 2014 update. Eur Urol 2015;67(5):913-24.

19. Escudero-Esparza A, Martin TA, Davies ML, Jiang WG. PGF isoforms, PLGF-1 and PGF-2, in colorectal cancer and the prognostic significance. Cancer Genomics Proteomics 2009;6(4):239-46.

20. Meng Q, Duan P, Li L, Miao Y. Expression of Placenta Growth Factor Is Associated with Unfavorable Prognosis of Advanced-Stage Serous Ovarian Cancer. Tohoku J Exp Med 2018;244(4):291-96.

21. Zhang L, Chen J, Ke Y, Mansel RE, Jiang WG. Expression of Placenta growth factor (PIGF) in non-small cell lung cancer (NSCLC) and the clinical and prognostic significance. World J Surg Oncol 2005;3:68.

22. Pompeo E, Albonici L, Doldo E, Orlandi A, Manzari V, Modesti A, Mineo TC. Placenta growth factor expression has prognostic value in malignant pleural mesothelioma. Ann Thorac Surg 2009;88(2):426-31. 